\title{
Pressure Ulcers and Malnutrition: Results from a Snapshot Sampling in a University Hospital
}

\author{
Georgia Tsaousi $^{\mathrm{a}}$ George Stavrou $^{\mathrm{b}}$ Aristidis loannidis $^{\mathrm{b}}$ Spyros Salonikidis ${ }^{\mathrm{b}}$ \\ Katerina Kotzampassi ${ }^{\text {b }}$
}

Departments of a Anesthesiology and Intensive Care Unit and b Surgery, AHEPA University Hospital, Faculty of Medicine, Aristotle University of Thessaloniki, Thessaloniki, Greece

\section{Key Words}

Pressure ulcers · Nutritional status · Malnutrition universal screening tool

\begin{abstract}
Objective: We aimed to ascertain the factors potentially contributing to the manifestation of pressure ulcers (PU) due to poor nutritional status in a nonselected hospitalized population. Subjects and Methods: This is a prospective cohort study of 471 adult inpatients treated at our university hospital. Study parameters included anthropometric data, demographics, medical history, mood status, diet-related factors and self-perception of health status. For each participant, the body mass index (BMI) was calculated, and a malnutrition universal screening tool (MUST) was used to screen for nutritional deficiencies, with the presence of PU constituting the outcome of interest. An independent-samples $t$ test, $x^{2}$ analysis and logistic regression analysis were performed. $\boldsymbol{R} \boldsymbol{e}-$ sults: The overall prevalence of PU in our cohort was $14.2 \%$. Advanced age, low BMI, poor health status by self-assessment, serious mood disorders, malnutrition, abnormal appetite status, a quantity of food intake half of normal, an artificial diet, limited or no autonomy in everyday activities $(p<0.01$ for all) and recent weight loss $(p<0.05)$ were iden-
\end{abstract}

tified as important determinants for the manifestation of PU. Multivariate analysis highlighted limited autonomy in everyday activities (OR 6.456 and 95\% Cl 3.212-12.973; $\mathrm{p}=0.000$ ), MUST score (OR 3.825 and 95\% Cl 1.730-8.455; $\mathrm{p}=0.001$ ) and artificial diet (OR 1.869 and 95\% Cl 1.247-2.802; $\mathrm{p}=$ 0.018 ) as the most powerful predictors of PU development. Conclusion: Poor nutritional status, limited autonomy in everyday activities and artificial nutrition seemed to confer noteworthy prognostic value regarding PU development in the acute-care setting.

(c) 2014 S. Karger AG, Basel

\section{Introduction}

Skin ulceration is considered to be one of the most challenging and physically debilitating clinical entities for nearly all health-care disciplines, with a reported prevalence ranging from 0.4 to $38 \%$, in acute-care settings [16]. Apart from the individual's discomfort, pressure ulcers (PU) augment the workload in all health-care sectors because of the associated delayed rehabilitation and prolonged hospitalization, which can eventually have an adverse secondary impact on health care or cause premature morbidity and mortality $[7,8]$. To further complicate the

\begin{tabular}{|c|c|}
\hline KARGER 125 & $\begin{array}{l}\text { (C) } 2014 \text { S. Karger AG, Basel } \\
1011-7571 / 14 / 0241-0011 \$ 39.50 / 0\end{array}$ \\
\hline $\begin{array}{l}\text { E-Mail karger@karger.con } \\
\text { www.karger.com/mpp }\end{array}$ & $\begin{array}{l}\text { This is an Open Access article licensed under the terms of the } \\
\text { Creative Commons Attribution-NonCommercial } 3.0 \text { Un- } \\
\text { ported license (CC BY-NC) (www.karger.com/OA-license), } \\
\text { applicable to the online version of the article only. Distribu- } \\
\text { tion permitted for non-commercial purposes only. }\end{array}$ \\
\hline
\end{tabular}

Katerina Kotzampassi, $\mathrm{MD}, \mathrm{PhD}$

Department of Surgery

AHEPA University Hospital

GR-54636 Thessaloniki (Greece)

E-Mail kakothe@yahoo.com 
issue, PU are multifactorial in origin, with both preventable and nonpreventable components; the significance of these factors is yet to be elucidated.

Malnutrition, an ordinary clinical feature in hospitalized populations, constitutes a potentially reversible predisposing factor for both ulcer formation and delayed wound-healing $[5,7,8]$. Nutritional deficiencies impede the normal processes that allow progression through the specific stages of wound-healing by prolonging the inflammatory phase, decreasing fibroblast proliferation and altering collagen synthesis, which attenuate wound tensile strength and promote infection $[9,10]$.

To date, the exact causal relationship between PU and nutritional status has not been fully clarified. Methodological shortcomings notwithstanding, cross-sectional and prospective studies suggest that there is a fairly strong interrelation between undernourishment and PU development $[1,3-6,8]$. However, a Cochrane review published in 2003 [11] failed to supply strong scientific evidence for a direct relationship between poor nutrition and the development of PU and healing [11].

More evidence is needed as to whether or not a relationship between PU and malnutrition exists, so we embarked on this study with a view to gaining a thorough insight into the factors that engender skin breakdown via poor nutritional status in a nonselected group of adult patients in a Greek university hospital.

\section{Subjects and Methods}

\section{Study Population}

This observational prospective study was conducted according to the Declaration of Helsinki guidelines and was approved by our Hospital Scientific Council. Written informed consent was obtained from all the subjects/patients, their families or legal representatives. We excluded patients being treated in an intensive care unit and those with a deteriorated level of consciousness who had no caregivers or proxies to provide the necessary information.

\section{Data Collection}

Six groups, each consisting of 2 designated health-care professionals (i.e. nurses, dieticians or doctors, one of whom worked in the patient's ward and the other independently) interviewed all heterogeneous adult patients at our university hospital or their family members, where available, and reviewed the consenting patients' medical charts for the purpose of completing a specifically prepared standardized questionnaire at a single-day audit. In our 680 -bed university hospital, a total of 603 inpatients were registered on the day of the audit. Considering both predefined exclusion criteria and missing data, 471 consecutive hospitalized patients were enrolled in the final analysis.

In particular, the following factors were registered: (1) demographic and anthropometric characteristics (age, gender, mea- sured weight, height, mid-arm and calf circumference), (2) hospitalization data (participating specialties, presurvey and total hospital stay), (3) quality of health status assessed according to the reported comorbidities and the participants' self-perceived adequacy of their health status and (4) dietary-related parameters (self-reported weight loss within the last 3 months, the extent of dependence in everyday activities, appetite status, mood disorders and quantity and type of nutritional intake during hospitalization).

The body mass index (BMI) was calculated for each participant. A malnutrition universal screening tool (MUST) was used to screen for nutritional deficiencies $[12,13]$. Patients were considered to be at high risk if they had a BMI $<18.5$, had experienced $>10 \%$ unintentional weight loss in the previous 3-6 months or had had no nutritional intake for $>5$ days. They were considered to be at moderate risk if they had a BMI $18.5-20.0$ or had experienced $5-10 \%$ weight loss in the previous 3-6 months. All other patients were classified as low-risk [13]. The presence of PU was also recorded and constituted the outcome of interest. All stage I-IV lesions, according to the European Pressure Ulcer Advisory Panel classification, were registered as PU [14]. Total length of stay (LOS) in hospital was used as an index for morbidity assessment.

A random check was conducted by G.T. and K.K. in order to eliminate errors in the data collection on paper sheets and transferring processes before the data were entered into a computer database.

\section{Statistical Analysis}

The normality of data was assessed by the KolmogorovSmirnov test. The Student t test was used for comparison of means of continuous variables and normal distributed data and the Mann-Whitney U test was applied in the case of non-normally and non-continuously distributed data. Categorical variables were assessed with the $\chi^{2}$ test or the Fisher exact test when the expected value of a cell was $<5$. Odds ratios (OR) with $95 \%$ confidence intervals (CI) were computed using univariate and multivariate stepwise logistic regression models with $\mathrm{PU}$ development as the response variable. For all statistical procedures, $\mathrm{p}<0.05$ was considered significant. Data were analyzed using the SPSS version 18.0 (SPSS Inc., Chicago, Ill., USA).

\section{Results}

The recorded prevalence of PU was $14.2 \%(n=67)$. The nutritional and hospitalization characteristics of the participants were stratified on the basis of PU development and are shown in table 1 . Individuals with PU manifestation featured with greater age, lower BMI $(<18)$, deteriorated clinical status according to the self-assessment, limited everyday activities (able to walk only with assistance or being bedfast) and moderate or serious mood disorders ( $\mathrm{p}<0.001$ for all). Comorbidities were present in 268 patients (56.9\%); the most common were diabetes mellitus in 95 patients ( $36.6 \%)$, followed by hypertension in $70(27.2 \%)$, stroke in $56(21.6 \%)$, chronic obstructive 
Table 1. Nutritional and hospitalization characteristics of participants according to the manifestation of PU

\begin{tabular}{lccc}
\hline Variable & Non-PU group & PU group & p value \\
\hline Patients & $404(85.8)$ & $67(14.2)$ & \\
Age, years & $62.5 \pm 17.3$ & $74.4 \pm 14.6$ & 0.001 \\
Female gender & $246(60.9)$ & $31(46.3)$ & 0.202 \\
Comorbidity & $221(54.7)$ & $47(70.2)$ & 0.190 \\
BMI <18.5 & $9(2.3)$ & $13(19.4)$ & 0.000 \\
Poor health status by self-assessment & $98(24.4)$ & $51(76.3)$ & 0.001 \\
Presence of mood disorders & $83(20.5)$ & $45(67.1)$ & 0.000 \\
Limited autonomy in everyday activities & $90(22.3)$ & $60(89.5)$ & 0.000 \\
MUST (undernourishment) & $253(62.6)$ & $63(94.5)$ & 0.000 \\
Recent weight loss & $246(60.9)$ & $21(31.3)$ & 0.041 \\
Abnormal appetite status & $108(26.7)$ & $42(62.7)$ & 0.002 \\
Quantity of food consumed in the last week & & & \\
$\quad$ (below normal) & $158(39.1)$ & $55(82.1)$ & 0.000 \\
Artificial diet & $21(5.3)$ & $27(40.2)$ & 0.000 \\
Arm perimeter $<22 \mathrm{~cm}$ & $36(12.1)$ & $12(17.9)$ & 0.675 \\
Calf perimeter <31 cm & $75(25.2)$ & $25(37.3)$ & 0.432 \\
Presurvey hospital LOS, days & $4.3(8.7)$ & $7.8(8.2)$ & 0.017 \\
Hospital LOS, days & $7.2 \pm 7.1$ & $16.1 \pm 6.6$ & 0.000 \\
\hline
\end{tabular}

Data are expressed as $\mathrm{n}(\%)$ or mean $\pm \mathrm{SD}$.

pulmonary disease in $27(10.5 \%)$ and cardiovascular disease in $10(4.1 \%)$. No significant difference between the 2 subgroups studied was identified $\left(\chi^{2}=8.61 ; \mathrm{p}=0.125\right)$.

When our cohort was classified according to the reason for admission into a pathological (i.e. involving general, neurological and cardiac pathologies) and a surgical subset, 45 (67.2\%) of the PU patients and 241 of the nonPU patients (59.6\%) involved pathological cases. No significant difference between groups was identified with regard to surgical cases $\left(\chi^{2}=1.08 ; \mathrm{p}=0.429\right)$.

With regard to nutrition-related parameters, the $\mathrm{PU}$ group presented with undernourishment (a risk for/documented malnutrition), diminished (less than normal) food intake during the last week $\left(\chi^{2}=12.07 ; p=0.002\right.$ and $\chi^{2}=27.43 ; \mathrm{p}=0.000$, respectively) and noticeable weight loss $(>5 \%)$ during the last 3 months $\left(\chi^{2}=11.38 ; \mathrm{p}=0.014\right)$. Common reasons for not eating were identified as loss of appetite in 99 cases $(46.5 \%)$, difficulty in chewing or swallowing in 8 (3.7\%) and nausea in 21 (9.8\%); loss of appetite was the predominant reason for not eating in the PU group $\left(\chi^{2}=9.12 ; \mathrm{p}=0.021\right)$.

Regular hospital food with no particular dietary plan was the source of nutrition for 269 (57.1\%) patients. One hundred and sixty-eight (35.7\%) were given hospital food modified for some form of special diet and 34 (7.1\%) were on enteral or total parenteral nutrition. An artificial diet (i.e. enteral or parenteral nutrition) was more frequently applied in the PU group than in the non-PU group $\left(\chi^{2}=\right.$ 32.67; $\mathrm{p}=0.000$ ).

The mean presurvey LOS and total hospital LOS were significantly prolonged in the PU group when compared to the non-PU group ( $\mathrm{t}$ test; $\mathrm{p}=0.017$ and $\mathrm{p}=0.000$, respectively). Almost 2 of 3 patients in the non-PU group had a hospital LOS of $<8$ days $(64.3 \% ; \mathrm{n}=260)$. The hospital LOS-dependent distribution of PU development differed significantly between hospital stay subgroups $\left(\chi^{2}=\right.$ 45.17; $\mathrm{p}=0.000$ ) with the incidence of PU development being proportional to prolonged hospital stay (fig. 1).

Univariate logistic regression analysis identified advanced age, low BMI, poor health status by self-assessment, serious mood disorders, malnutrition assessed by MUST, recent weight loss, abnormal appetite status, quantity of food intake $<50 \%$, artificial diet (enteral or total parenteral nutrition) and limited or no autonomy in everyday activities as important determinants for manifestation of PU (table 2).

By entering the nutrition status-related variables into a stepwise logistic regression model, limited autonomy $[\beta=1.865$; standard error $(\mathrm{SE})=0.356$; OR 6.456 and 95\% CI 3.212-12.973; $\mathrm{p}=0.000]$, MUST score $(\beta=1.341$; $\mathrm{SE}=0.405$; OR 3.825 and $95 \%$ CI $1.730-8.455 ; \mathrm{p}=0.001)$ and artificial diet $(\beta=0.625$; SE $=0.207$; OR 1.869 and $95 \%$ CI $1.247-2.802 ; \mathrm{p}=0.018$ ) were highlighted as the most powerful predictors of PU development. 


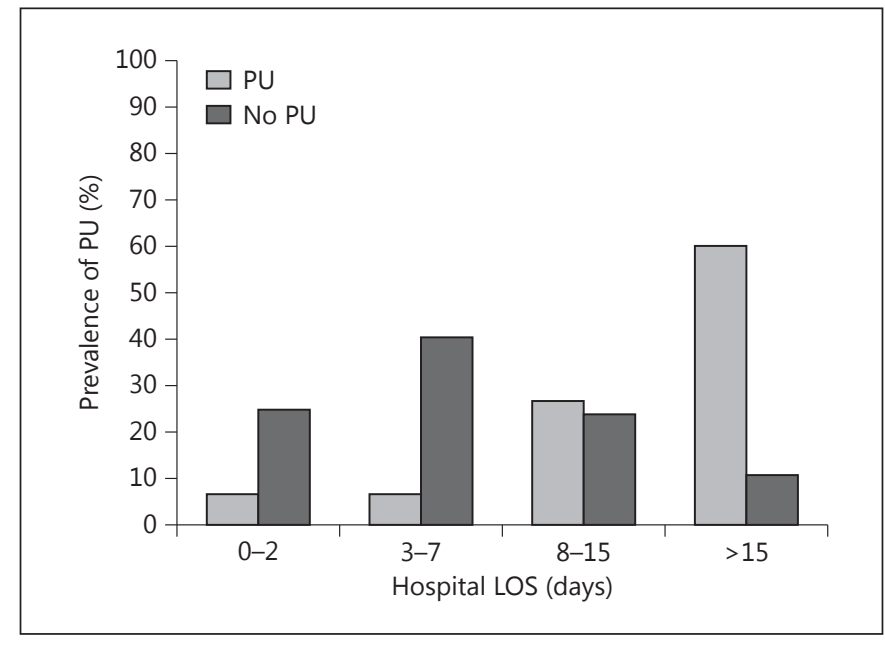

Fig. 1. Presence of PU stratified to hospital LOS groups.

\section{Discussion}

This study showed the association between undernutrition (assessed by MUST) or nutrition-related parameters (e.g. advanced age, low BMI, poor health status, serious mood disorders, impaired appetite, a food intake of $<50 \%$ during the last week, self-reported weight loss during the last 3 months, limited or no autonomy in everyday activities and artificial nutrition) and hospitalization-related parameters (such as presurvey and total hospital LOS) with the presence of PU in an acute-care setting. Among these, malnutrition-limited autonomy in everyday activities and artificial diet were identified as major contributors to PU development.

Research has shown that inadequate nutrition is frequently associated with the loss of the cushioning effect of fat mass over the bony prominences, physical weakness, dehydration and edema; it also weakens skin resistance, mobility and the immune defense, all of which seem to be predisposed for the development of PU [3, 4, 5, 8-10]. A recent, multicenter, cross-sectional audit in an acute-care facility in Australia [3] reported an overall adjusted OR of 2.6 of having a PU when malnourished as increasing from approximately 2 -fold with moderate malnutrition to nearly 5-fold with severe malnutrition. In our clinical setting, nutritional deficiencies were associated with a higher risk for PU of almost 4 -fold. The OR increased dramatically, along with the severity of nutrition deficiency, from approximately 3 -fold for patients at risk of malnutrition to almost 7 -fold for those with severe malnutrition. A novel finding of our study was that the
Table 2. Univariate logistic regression analysis of the association between factors related to nutrition status and the development of PU

\begin{tabular}{|c|c|c|}
\hline Variable & OR $(95 \% \mathrm{CI})$ & $\mathrm{p}$ value \\
\hline Age & $1.056(1.022-1.091)$ & 0.003 \\
\hline \multicolumn{3}{|l|}{ BMI } \\
\hline $18.5-28$ & 1.0 & ref. \\
\hline$<18.5$ & $7.893(1.783-28.932)$ & 0.003 \\
\hline$>28$ & $2.861(1.068-8.458)$ & 0.047 \\
\hline \multicolumn{3}{|l|}{ Self-assessment of health } \\
\hline status & $2.585(1.329-5.031)$ & 0.005 \\
\hline \multicolumn{3}{|l|}{ Mood disorders } \\
\hline None & 1.0 & ref. \\
\hline Moderate & $1.167(0.294-4.634)$ & 0.827 \\
\hline Serious & $8.520(2.117-24.289)$ & 0.003 \\
\hline \multicolumn{3}{|c|}{ Autonomy in everyday activities } \\
\hline \multicolumn{3}{|l|}{ Able to walk with no } \\
\hline assistance & 1.0 & ref. \\
\hline \multicolumn{3}{|l|}{ Able to walk only with } \\
\hline assistance & $6.981(1.421-24.297)$ & 0.017 \\
\hline Bedridden & $17.212(9.283-46.562)$ & 0.000 \\
\hline \multicolumn{3}{|l|}{ MUST } \\
\hline Well-nourished & 1.0 & ref. \\
\hline At risk of malnutrition & $3.398(1.209-9.552)$ & 0.020 \\
\hline Malnourished & $7.013(2.152-23.506)$ & 0.007 \\
\hline Recent weight loss & $2.356(1.097-5.721)$ & 0.027 \\
\hline Abnormal appetite status & $5.500(1.815-16.669)$ & 0.003 \\
\hline \multicolumn{3}{|c|}{ Quantity of food consumed in the last week } \\
\hline Normal & 1.0 & ref. \\
\hline Less than normal & $1.077(0.670-2.828)$ & 0.612 \\
\hline Half of normal & $1.761(0.509-6.091)$ & 0.132 \\
\hline Quarter of normal & $12.043(2.952-29.137)$ & 0.001 \\
\hline \multicolumn{3}{|l|}{ Dietary type } \\
\hline Hospital food & 1.0 & ref. \\
\hline Specific diet & $1.333(0.704-2.686)$ & 0.302 \\
\hline Enteral & $2.629(1.474-14.257)$ & 0.008 \\
\hline Total parenteral nutrition & $10.867(1.626-27.190)$ & 0.000 \\
\hline
\end{tabular}

MUST score was identified as a strong indicator of the risk for PU development, which further reinforces its wide implementation for a valid assessment of nutritional status.

Current guidelines recommend universal nutritional screening for all patients with/at risk of developing skin ulceration upon initial assessment or with any change in condition [8]. Several tools have been suggested to assess nutritional status in an average hospital population. Each has its own strengths and weaknesses, so nutritional status should not be assessed using only one tool or marker. A number of divergent factors related to inadequate food intake, unintentional weight loss and nutritional deficiencies, e.g. functional and psychosocial causes, should
Tsaousi/Stavrou/Ioannidis/Salonikidis/ Kotzampassi 
also be considered. Obtaining insight into predisposing factors for the development of PU should make it possible to identify the subgroups of patients who are at risk.

Nutrition-related factors that are considerably linked to PU development or impaired healing include advanced age, reduced food and fluid intake, chewing and swallowing difficulties and an impaired ability to eat independently. These can all result in low BMI and unintentional weight loss $[3,4,8,15-18]$.

In a large, case-control study, Fogerty et al. [15] reviewed admission and discharge data from over 6 million subjects in acute-care hospital settings in order to identify risk factors and demographic differences between those who developed PU and those who did not. Interestingly, an age of over 75 years emerged as the strongest PU risk factor, with an OR of 12.63. Greater age is associated with an increased risk of malnutrition, as disease prevalence in this group increases and body composition changes [16]. In our analysis, the difference in mean age between subsets with or without PU was clear (74.4 and 62.5 years, respectively), a profile that indeed points to a heightened risk in an aged population.

Although BMI can be affected by the fluid status of an individual and the accuracy of its calculation depends on the investigator's level of experience, it is still considered an objective measurement for the determination of nutritional status and constitutes a pivotal component of several malnutrition screening tools $[11,12]$. Several studies have identified low BMI as a contributing factor to the development of PU [4, 16-18].

Poor nutritional intake and unintentional weight loss were strongly related to the manifestation of PU in our hospital setting, further reinforcing previous findings that have identified inadequate nutritional intake and recent weight loss as independent risk factors for PU, with the reported OR averaging 4.6 and 2.3 , respectively $[1,4$, 19-21]. In accordance with our findings, a recent crosssectional study reported that $\mathrm{BMI}<18.5$, unintentional weight loss, poor nutritional intake and being bedfast were strongly related to the presence of PU in hospitalized patients, with the highest predictive value being attributed to being bedfast (OR 22.96) [4]. In our cohort, bedridden patients presented with a 17 -fold higher prevalence for the manifestation of PU, while limited autonomy was highlighted as the variable with the highest predictive value for PU development. The autonomy component is identified as a key factor because limited autonomy in everyday activities interacts with weight loss and nutritional intake for PU development. Weight loss and nutritional intake are related to limited autonomy because these malnutrition parameters influence an individual's functional capacity $[4,16]$.

The role of nutritional support in the wound-healing process remains unclear [5]. Interestingly, our multivariate analysis showed that artificial diet was an independent predictor of the development of PU. The possible explanation for this finding is 2-dimensional, indicating either a more advanced deterioration in the health status of patients receiving an artificial diet or else a higher nutritional quality of the normal or specific diet in our hospital setting.

Finally, it is not surprising that depressed mood emerged as a risk factor for the development of PU, with much evidence to support the pivotal role of mood disorders in undernourishment, in close association with clinical and functional status deterioration [22].

As has already been pointed out, a substantial body of evidence supports that the development of PU incurs greater hospitalization costs and is related to a longer hospital LOS and greater utilization of hospital resources [1, $3,4,7,8]$. Most published data converge on a prolongation of hospital LOS of 6-8 days, on average, among patients with/without PU; this is relatively consistent with the hospital LOS in our PU group $[6,19,23]$.

Although the screening of patients at risk of developing skin breakdown is considered an essential initial step in the structured process of a comprehensive care plan for the prevention and treatment of PU, it is often overlooked and underrecognized in hospitalized patients $[8,16]$. Traditionally, the development of PU has been viewed as a failure of care, and the rate of occurrence is thought to be a key quality indicator. A general comparison of PU prevalence in our cohort (14.2\%) revealed it to be within the upper limit of the reported range (7.1-15.2\%) for in-hospital patients [1-4]. This could be attributed to the selection criteria applied, as the PU group in our cohort involved patients even from stage I, which is often excluded from the final analysis. Unfortunately, meaningful comparisons between prevalence and incidence rates across studies cannot always be made due to the different grading systems for PU and different methods of data collection used, different (or lacking) population characteristics and different inclusion and exclusion criteria.

We acknowledge the limitations of this study. Our single-center population was heterogeneous, incorporating patients from different age groups. It also involved a rather small sample. Furthermore, no evidence was provided with regard to outcome-relevant end points and the impact of dietary interventions on the clinical course of individuals with PU. 


\section{Conclusion}

Our findings reveal that poor nutritional status, limited autonomy in everyday activities and artificial nutrition confer noteworthy prognostic value regarding the development of PU in acute-care settings. The economic consequence of this health outcome index is substantial, as the hospital LOS was found to be 2 times longer for patients with PU. Malnutrition is a reversible risk factor for the manifestation of PU. Our results emphasize the need for constant awareness of providers of medical care in Greek hospitals concerning the issue of malnutrition as well as the importance of the implementation of adequate nutritional care for individuals prone to $\mathrm{PU}$ across the continuum of care.

\section{References}

1 Fisher AR, Wells G, Harrison MB: Factors associated with pressure ulcers in adults in acute care hospitals. Adv Skin Wound Care 2004; 17:80-90.

$>2$ Schoonhoven L, Grobbee DE, Donders AR, et al: Prediction of pressure ulcer development in hospitalized patients: a tool for risk assessment. Qual Safety Health Care 2006;15:6570.

3 Banks M, Bauer J, Graves N, et al: Malnutrition and pressure ulcer risk in adults in Australian health care facilities. Nutrition 2010; 26:896-901.

4 Shahin ES, Meijers JM, Schols JM, et al: The relationship between malnutrition parameters and pressure ulcers in hospitals and nursing homes. Nutrition 2010;26:886-889.

$>5$ Stratton RJ, Ek AC, Engfer M, et al: Enteral nutritional support in prevention and treatment of pressure ulcers: a systematic review and meta-analysis. Ageing Res Rev 2005;4: 422-450.

6 Lyder CH, Wang Y, Metersky M, et al: Hospital-acquired pressure ulcers: results from the National Medicare Patient Safety Monitoring System Study. J Am Geriatr Soc 2012;60: 1603-1608.

7 Little MO: Nutrition and skin ulcers. Curr Opin Clin Nutr Metab Care 2013;16:39-49.

-8 Dorner B, Posthauer ME, Thomas D: The role of nutrition in pressure ulcer prevention and treatment: National Pressure Ulcer Advisory Panel white paper. Adv Skin Wound Care 2009;22:212-221.
9 Campos ACL, Groth AK, Branco A: Assessment and nutritional aspects of wound healing. Curr Opin Clin Nutr Metab Care 2008; 11:281-288.

10 Kolios G, Kotzampassi K, Manousou P, et al: Enteral nutrition affects nitric oxide produc tion in peripheral blood and liver after a postoperative lipopolysaccharide-induced endotoxemia in rats. Nutrition 2007;23:575-581.

11 Langer G, Schloemer G, Knerr A, et al: Nutrition for preventing and treating pressure ulcers (review). The Cochrane Library 2005; (issue 4).

12 Prospective Studies Collaboration; Whitlock G, Lewington S, et al: Body mass index and cause-specific mortality in 900,000 adults: collaborative analyses of 57 prospective studies. Lancet 2009;373:1083-1096.

13 Stratton RJ, Hackston A, Longmore D, et al: Malnutrition in hospital outpatients and inpatients: prevalence, concurrent validity and ease of use of the 'malnutrition universal screening tool' ('MUST') for adults. Br J Nutr 2004;92:799-808.

14 EPUAP (European Pressure Ulcer Advisory Panel): Pressure ulcer treatment guidelines 2004. http:www.epuap.org/gltreatment.html

15 Fogerty MD, Abumrad NN, Nanney L, et al: Risk factors for pressure ulcers in acute care hospitals. Wound Rep Regen 2008;16:11-18.
16 Tsaousi G, Panidis S, Stavrou G, et al: Prognostic indices of poor nutritional status and their impact on prolonged hospital stay in a Greek university hospital. Biomed Res Int 2014;2014:924270.

17 Kottner J, Gefen A, Lahmann N: Weight and pressure ulcer occurrence: a secondary data analysis. Int J Nurs Stud 2011;48:1339-1348.

18 VanGilder C, MacFarlane G, Meyer S, et al: Body mass index, weight, and pressure ulcer prevalence: an analysis of the 2006-2007 International Pressure Ulcer Prevalence Surveys. J Nurs Care Qual 2009;24:127-135.

19 Cowan LJ, Stechmiller JK, Rowe M, et al: Enhancing Braden pressure ulcer risk assessment in acutely ill adult veterans. Wound Repair Regen 2012;20:137-148.

20 Guenter P, Malyszek R, Bliss DZ, et al: Survey of nutritional status in newly hospitalized patients with stage III or stage IV pressure ulcers. Adv Skin Wound Care 2000;13:164-168.

21 Mathus-Vliegen EMH: Nutritional status, nutrition and pressure ulcers. Nutr Clin Pract 2001;16:286-291.

22 Ahmed S, Leurent B, Sampson EL: Risk factors for incident delirium among older people in acute hospital medical units: a systematic review and meta-analysis. Age Ageing 2014; 43:326-333.

23 Allman RM, Goode PS, Burst N, et al: Pressure ulcers, hospital complications, and disease severity: impact on hospital costs and length of stay. Adv Wound Care 1999;12:2230 . 